\title{
Molecular and cell biology in clinical medicine: Introduction
}

\author{
DAVID WEATHERALL
}

The past few years have seen a revolution in the biological sciences comparable to that in physics earlier this century. It is already clear that the remarkable achievements of molecular and cell biology have enormous potential for medical research and practice and that the medical sciences are about to enter the most exciting period of their development.

Over the next few months the $B M \mathcal{F}$ will carry a series of articles that outline some of these new developments and their application to the study of human disease. Although for convenience of presentation they will appear in two parts, one covering molecular biology and the other cell biology, it will become apparent that the two fields are inseparable; molecular biology describes the anatomy and organisation of the molecules of living organisms and modern cell biology encompasses the way in which they work together as an orchestrated whole to mediate and regulate cellular function.

By way of an introduction to this important and timely series I shall summarise briefly the clinical relevance of some of the ground that will be covered.

\section{Some applications of molecular biology in clinical medicine}

The extraordinary technical developments in molecular biology during the past few years have provided us with the tools to construct libraries of cloned deoxyribonucleic acid (DNA) representing most of the human genome, to isolate and determine the structure of human genes, and to develop a variety of ingenious systems for studying gene function in the test tube, in cell culture, and even in intact animals. Any gene that is worth studying can now

Nuffield Department of Clinical Medicine, University of Oxford, John Radcliffe Hospital, Oxford OX3 9DU

SIR DAVID WEATHERALL, MD, FRS, professor of medicine be isolated, and the idea of a complete map of the human genome is no longer a pipe dream.

The most immediate medical application of recombinant DNA technology has been in clinical genetics, where it has been possible to work out the molecular pathology of many genetic diseases and to institute new techniques for detection of carriers and prenatal diagnosis. It is interesting to reflect that it is less than 10 years since the first human gene was cloned and sequenced, yet we already have a good idea of the repertoire of mutations that underlie single gene disorders. Perhaps one of the most spectacular successes has been the development of methods to track down genes for genetic diseases of completely unknown aetiology and, by what is now called reverse genetics, to work out what these genes do and hence to determine the cause of the particular disease. Within the next few years gene therapy probably will be established, at least for a few important genetic disorders.

It is perhaps in the study of the common illnesses of Western society such as degenerative vascular disease, diabetes, major psychiatric conditions, cancer, and rheumatic disorders that molecular genetics will, in the long term, play its most important part. Although major advances in epidemiology have given us some valuable information about the factors that contribute to the high incidence of some of these conditions, hitherto their management has been largely symptomatic. The application of molecular biology could completely change our approach to these intractable problems.

It is not immediately obvious how molecular genetics could help us to understand the pathogenesis of the changes in the vessel wall that give rise to coronary artery disease and stroke. After all, epidemiologists have told us that risk factors such as diet, smoking, hypertension, and obesity play a major part in their development; genetic factors seem to be of secondary importance. But studies of the molecular genetics of uncommon disorders with a high incidence of coronary atheroma - familial hypercholesterolaemia, for example -have provided us with insights into a number of genes that may be implicated in the pathology of degenerative vascular disease. An understanding of the function (and malfunction) of several well defined candidate genes in such a polygenic system should lead us to 
a better understanding of the molecular pathology of atherosclerosis and hence to its better management. The same goes for other common disorders. Recent work indicates that susceptibility to type I diabetes may reflect a single amino acid difference in one of the products of the class II genes of the HLA-DR system. As we come to characterise other HLA related conditions at the molecular level we should be in a better position to determine the environmental agents that act on individuals with such a precisely defined genetic susceptibility.

We can extend this argument to cover common psychiatric disorders. Although it is unusual for schizophrenia or the bipolar affective disorders to segregate within families as though they were the result of single mutant genes, such families do exist. By means of the new techniques of molecular genetics we shall probably be able to define and characterise the genes concerned. This in turn may lead us to a much better understanding of the neuropathology and neurochemistry of these conditions in general.

There are strong hints of the diagnostic and therapeutic possibilities of molecular biology. Cloned genes are already finding wide application in the diagnosis of bacterial, viral, and parasitic illnesses, and probes for oncogenes and immunoglobulin and $\mathrm{T}$ cell receptor genes are being used to type tumours. Several human genes have been persuaded to synthesise their products in bacterial systems. The correction of the anaemia of renal failure by genetically engineered erythropoietin is a good example of an early success in the rapidly expanding biotechnology industry. Numerous other therapeutic and diagnostic agents will follow. For example, genetically engineered factors VIII and IX, a variety of hormones, and tissue plasminogen activator will soon be available for clinical trials. The problem of antibiotic resistance is being approached by producing hybrid antibiotics, constructed by altering the genes responsible for antibiotic production in fungi and bacteria. Recombinant DNA technology offers new approaches to vaccine production-for example, augmenting vaccinia virus with genes from other viruses such as rabies and the production of large quantities of specific antigens from malarial parasites. The discovery of the retrovirus that causes the acquired immune deficiency syndrome (AIDS) so soon after this condition was first recognised exemplifies the strength of this new technology; if a means to control AIDS is found it is likely to come from the same discipline.

\section{Amalgamation of molecular and cellular biology}

Although less newsworthy, advances in our understanding of cell biology have been almost as rapid as those in molecular biology. This subject has equal potential for medical application, particularly for understanding cancer, autoimmune disease, human development, and the genesis of congenital malformation.

The application of recombinant DNA technology to the problems of cell biology are starting to unravel some of the mysteries of cell behaviour. For example, in many tissues there are pluripotential stem cells that go through cycles of multiplication before entering terminal differentiation towards their specialised functions. It is becoming possible to define specific genes implicated in the regulation of different phases of the cell cycle and in the critical steps of commitment and differentiation. The availability of gene probes and methods of studying the physical properties of inactive and active genes is enabling many of the processes of proliferation and differentiation to be described at the molecular level.

In understanding the orchestration of cellular proliferation and function it is important to learn how cells respond to external regulatory signals such as hormones and growth factors. Considerable progress has been made in relating the action of external regulatory molecules to the cyclic AMP, inositol triphosphate, and phospholipid dependent protein kinase $C$ messenger systems. Equally important advances have been made in purifying some of the growth factors and other regulatory proteins that control cell proliferation. Once their structure is known their genes can be isolated and, by recombinant DNA technology, used to produce large quantities of their products for determining how they work and for assessing their clinical potential. A whole family of haemopoietic growth factors has been obtained in this way, many of which are already available for analysing how they regulate marrow progenitors and for use in clinical trials.

An understanding of what controls cell proliferation and differentiation has important applications for clinical practice. For example, the past few years have seen an enormous expansion of the application of the combined techniques of molecular and cell biology to the study of cancer. The discovery of oncogenes, genes that are normally concerned with a variety of cellular housekeeping activities, and their abnormal function in cancer has opened up a whole new area of investigation into the basic processes of malignant transformation. The finding that abnormal activation of these genes is often related to the chromosomal changes that are found in many common cancers has brought together cell biology and cytogenetics in an exciting new area of cancer research. The message from these studies is that cancer is a diverse disorder resulting from genetic damage of many kinds, including recessive or dominant mutations, major DNA rearrangements, and point mutations, all of which may disturb the expression or biochemical function of the affected genes. We now appear to have the tools with which to unravel these processes.

Studies of cell membrane and receptor function promise to have equally important clinical applications. For example, recent work on the lifestyle of the low density lipoprotein receptor, including its internalisation and recycling and how these are regulated, has helped to define the many stages at which cholesterol metabolism might be altered and offered us new possibilities for the pharmacological control of cholesterol concentrations. By means of monoclonal antibody and protein engineering technology, it will probably be possible to modify receptor function. The isolation of the $T$ cell receptor and studies of the cell biology of antigen recognition have opened up a completely new approach to sorting out the mechanisms of the autoimmune processes that may underlie many common diseases. The discovery and characterisation of the "supergene" family that regulates the immune system and the lymphokines that constitute its cellular regulatory molecules are another remarkable example of the combined strengths of cell and molecular biology and their potential for the medical sciences.

\section{The future}

It is hoped that this series of short articles will give clinicians some insight into the way in which the medical sciences may be moving over the next few years. In effect, we appear to be changing from the era of whole patient physiology and pathology to the study of disease at the molecular and cellular level. This will undoubtedly alter the pattern of medical research. One effect will be to cloud the current compartmentalisation of medicine into watertight specialities; in the future cardiologists, nephrologists, and so on will be using the same techniques of cell and molecular biology to study diseases particular to their subject. It therefore follows that we may have to revise the way that research departments and resources are organised if we are to take full advantage of what recombinant DNA technology has to offer.

Clinicians may be worried about this reductionist approach to the study of disease, particularly at a time when we are being accused of ignoring the broader pastoral aspects of patient care and when there is a cry for a return to holistic medicine-that is, to rediscover how to be a good doctor. But there is no reason why a proper understanding of disease processes at the molecular and cellular level should have a deleterious effect on clinical care or on doctors' attitudes to their patients. On the contrary, in the long term it should enable us to treat many diseases more rationally and hence to reduce the proliferation of high technology medicine that characterises much of the current medical scene. But this will not happen overnight; there is likely to be a long period of development and evaluation of our new technology before its clinical application can be fully assessed. There may well be disappointments, but in the long term these new developments are likely to be of enormous benefit. 
All this will require some rethinking of medical education and the way in which our clinical schools and postgraduate programmes are organised in the future; the profession will have to take on board and accommodate individuals with a much broader range of scientific skills than has been necessary hitherto. This will require some urgent thought by those who are concerned with training our doctors in the future. I hope that this new series will help to provide some of the background information on which we can develop an informed debate on how these changes might be made while, at the same time, maintaining high standards of patient care.

\title{
Contemporary Themes
}

\section{Phobias and their management}

\author{
ISAAC MARKS， JOHN HORDER
}

\begin{abstract}
Disabling phobias and phobia like compulsive rituals are surprisingly common in the general population, though only a minority ask for help. Behavioural treatment (exposure) and antidepressants are the most helpful approaches. Most patients can help themselves if they use a self exposure approach systematically under the guidance of a clinician. The method seems well suited for use by general practitioners. Antidepressants are a useful adjuvant, not a substitute, for exposure when there is evidence of depression complicating the phobias or rituals.
\end{abstract}

\section{Introduction}

Phobias are fears of particular situations that do not normally bother most people. People with phobias tend to avoid the feared situations. Such phobic avoidance may cause considerable handicap. The patient knows that the fear is unreasonable, given the lack of objective danger. Phobias are distinguished from the normal fears that most people have by the intensity of the anxiety and the urge to avoid. The most common phobias are of public places (agoraphobia), of doing things under social scrutiny, and of specific cues like enclosed spaces, heights, darkness, thunderstorms, and particular types of animals and insects.

A related syndrome is obsessive-compulsive disorder. The anxious phobic avoidance in this syndrome closely resembles that in phobic disorders except that the frightening avoided situations are different. ${ }^{1}$ Obsessive-compulsive phobias are not of the public places feared by agoraphobics, the social cues that frighten social phobics, or the heights, darkness, thunder, animals, or other cues of panic in specific phobias. Instead, patients with this disorder have a phobia of imagined dirt, infection, or harming other people and

\footnotetext{
Institute of Psychiatry and Bethlem-Maudsley Hospital, De Crespigny Park, Denmark Hill, London SE5 8AF

ISAAC MARKS, MD, FRCPSYCH, professor of experimental psychopathology

Royal Free Hospital Medical School, London NW3

JOHN HORDER, FRCPSYCH, visiting professor

Correspondence to: Professor Marks.
}

avoid "contaminating" or "harmful" situations. Unlike in phobic disorder, obsessive-compulsive patients usually also engage in endless rituals such as washing and checking. These are usually done to reduce the fear or to avoid the cues that provoke the fears and rituals.

The results of recent epidemiological studies have shown that phobias and obsessive-compulsive disorder are surprisingly common. In the average general practice population of 2000 there are likely to be roughly 100 people with disabling phobias or obsessive-compulsive disorder. ${ }^{2}$ Only a minority of them ask for help despite effective treatment being available in recent years. More women than men tend to be sufferers, most phobias begin in young adult life, and atl social classes are affected about equally.

Almost every psychiatric treatment has been used for phobias, though few have been shown to be effective. In more than 100 carefully controlled studies in Europe and North America (reviewed by Marks, ${ }^{1}$ chapters 14-16), the best evidence of lasting help was for the behavioural treatment called exposure (other names for its various forms include habituation, desensitisation, and flooding). Exposure treatment helped phobics and ritualisers whether it was carried out in outpatient departments, general practitioners' surgeries, ${ }^{34}$ or at home ${ }^{5}$ and whether it was given by doctors, psychologists, nurses, ${ }^{3}$ or the patients themselves instructed by a self help manual,,$^{5-7}$ or computer. ${ }^{7}$ Most phobics and ritualisers find exposure therapy acceptable. Despite previous chronic disability about $85 \%$ of patients who start exposure treatment complete it; two thirds or more of those obtain moderate or appreciable relief of avoidance and panic-and of rituals in obsessive-compulsive disorder. ${ }^{1}$

Certain drugs, especially antidepressants, also help some types of phobia. Drugs are easy to prescribe and are part of the traditional medical role. Antidepressants take several weeks to act and have appreciable side effects; moreover, relapse often occurs when the drugs are stopped' so patients might have to take drugs for years. That is a drawback in syndromes such as those of anxious avoidance, which can last for decades. High doses of benzodiazepines give some relief in hours, but the effect evaporates even more quickly on stopping the drugs so there is a strong risk of drug dependence.

In contrast, exposure treatment is relatively new, and may at first sight seem more complicated than drugs and to need specialist staff. In fact exposure treatment can be fairly simple to prescribe and monitor for motivated patients yet be enduringly effective ${ }^{6}$-in six follow up studies of four to seven years cohorts maintained their gains. ${ }^{15}$ Its main drawback is that the patient has to work hard and systematically over weeks or months to achieve reliable gains. 